\title{
BMJ Open The role of academic health centres in building equitable health systems: a systematic review protocol
}

\author{
Alexandra Edelman, ${ }^{1,2}$ Judy Taylor, $^{3}$ Pavel V Ovseiko, ${ }^{4}$ Stephanie M Topp ${ }^{1}$
}

To cite: Edelman A, Taylor J, Ovseiko PV, et al. The role of academic health centres in building equitable health systems: a systematic review protocol. BMJ Open 2017;7:e015435. doi:10.1136/ bmjopen-2016-015435

- Prepublication history and additional material is available. To view please visit the journal (http://dx.doi.org/10.1136/ bmjopen-2016-015435)

Received 4 December 2016 Revised 21 February 2017 Accepted 22 March 2017

\section{(a) CrossMark}

${ }^{1}$ College of Public Health, Medical and Veterinary Sciences, Division of Tropical Health and Medicine, James Cook University, Queensland, Australia

${ }^{2}$ Tropical Australian Academic Health Centre, Queensland, Australia

${ }^{3}$ College of Medicine and Dentistry, James Cook University, Queensland, Australia ${ }^{4}$ Radcliffe Department of Medicine, Medical Sciences Division, University of Oxford, John Radcliffe Hospital, Oxford, UK

Correspondence to Ms Alexandra Edelman; alexandra.edelman@jcu.edu.au

\begin{abstract}
Introduction Academic health centres (AHCs) are complex organisations often defined by their 'tripartite' mission: to achieve high standards of clinical care, undertake clinical and laboratory research and educate health professionals. In the last decade, AHCs have moved away from what was a dominant focus on high impact (clinical) interventions for individuals, towards a more population-oriented paradigm requiring networked institutions and responsiveness to a range of issues including distribution of health outcomes and health determinants. Reflective of this paradigm shift is a growing interest in the role of AHCs in addressing health disparities and improving health system equity. This protocol outlines a systematic review that seeks to synthesise and critically appraise the current state of evidence on the role of AHCs in contributing to equitable health systems locally and globally.

Methods and analysis Electronic searches will be conducted on a pilot list of bibliographic databases, including Google Scholar, Scopus, MEDLINE, Psyclnfo, CINAHL, ERIC, ProQuest Dissertations \& Theses, Cochrane Library, Evidence Based Medicine Reviews, Campbell Library and A+Education, from 1 January 2000 to 31 December 2016. Apart from studies reporting clinical

Strengths and limitations of this study

- This will be one of the first attempts to synthesise and critically appraise evidence on the role of academic health centres in contributing to equitable health systems locally and globally.

- The systematic review protocol is developed using the Preferred Reporting Items for Systematic Reviews and Meta-analyses for Protocols (PRISMA-P) guidelines, with reference to the PRISMA-Equity 2012 Extension.

- Included studies will be assessed for methodological quality using the relevant Joanna Briggs Institute critical appraisal tools.

- The quality of synthesised evidence will be limited by the study designs of included studies, which are likely to be mostly observational studies, and limiting the review to published papers only will omit unpublished documentation of relevance to the review questions.

- Limiting searches to the literature published in the English language may lead to the omission of studies from non-English speaking countries.
\end{abstract} interventions or trials, all types of published peer-reviewed and grey literature will be included in the review. The single screening method will be employed in selecting studies, with two additional reviewers consulted where allocation is unclear. Quality and relevance appraisal utilising Joanna Briggs Institute critical appraisal tools will follow data extraction to a preprepared template. Thematic synthesis will be undertaken to develop descriptive themes and inform analysis.

Ethics and dissemination As the review is focused on the analysis of secondary data, it does not require ethics approval. The results of the study will be disseminated through articles in peer-reviewed journals and trade publications as well as presentations at relevant national and international conferences. Results will be further disseminated through networks and associations of AHCs. Protocol registration International Prospective Register for Systematic Reviews (PROSPER0) number CRD42016051802.

\section{INTRODUCTION}

Academic health centres (AHCs) are complex organisations that are largely defined by their 'tripartite' mission: to achieve high standards of clinical care, undertake clinical and laboratory research and educate health professionals. ${ }^{1}$ To deliver this mission, AHCs combine accredited higher education institutions delivering medical and other health professional education with one or more affiliated or owned teaching hospitals or health systems, ${ }^{2}$ employing a wide variety of governance and operational models. ${ }^{34}$ AHCs are well established in the United States and are either established or newly developing in a number of other high-income countries worldwide. ${ }^{15-7}$ Establishment of AHCs within Australia has commenced within recent years, ${ }^{8}$ including initiation of government-driven AHC designation processes for both metropolitan and regional models.

A recent scoping review of international literature focussed on AHCs demonstrated that a high proportion ( $>90 \%$ of those reviewed) of included studies focus on the Northern American context due to historical usage of the term 'AHC' in those countries. ${ }^{1}$ Another key finding 
of the review was that there is a growing focus of AHCs on the need to respond to so-called 'external' challenges ${ }^{1}$ such as the healthcare reform 'triple aim' of improving the health of individuals and populations while controlling healthcare costs. ${ }^{9}$ This 'external' focus represents a 'transformation' of AHCs in the United States, ${ }^{10} 11$ away from a traditional focus on high-impact interventions for individuals with serious disease ${ }^{12}$ and service integration in large urban hospitals and elite centres in capital cities, ${ }^{13}$ towards a population-oriented paradigm encompassing the three domains of patient care, education and research. ${ }^{14}$

A shift in AHCs' focus is also reflected in a growing interest among expert commentators in the role of AHCs in global health, ${ }^{15}$ including the need to develop 'broad, inexpensive and preventive treatment strategies among populations'rather than the creation of 'novel drugs, devices and other technologies', which is the traditional domain of AHCs. ${ }^{16}$ Interest in the potential for AHCs to develop "community-engaged' research agendas, which seek to address health disparities and uneven access to healthcare through better community engagement, ${ }^{17}$ and in the capacity of AHCs to develop innovative approaches to health workforce challenges, ${ }^{18}$ also attest to this new paradigm. A corresponding terminology change has been proposed-from academic health 'centres', implying healthcare in a single location, to academic health 'systems', reflecting the integrated, networked models of healthcare needed to meet new healthcare objectives. ${ }^{16} 19$

The perceived role and capacity of AHCs to drive progress towards broader healthcare goals, in the context of persisting global health disparities, has led some commentators to call for AHCs to 'accept responsibility' for prioritising and investing in research and innovation that benefit populations, including the communities where AHCs are located. ${ }^{15}$ Indeed, as the locus for health professional training and as organisations uniquely capable of generating and translating evidence and testing interventions, AHCs are seen by some as being particularly well situated to lead initiatives to improve care for vulnerable populations. ${ }^{20}$ Activities in service to this responsibility have been suggested and include new approaches to scaling up best practice, fostering multidimensional research platforms involving consideration of the social determinants of health and including cross-cultural competence and interprofessional education in AHC curricula. ${ }^{20}$

The interest of expert commentators in the capacity of AHCs to address health disparaties locally, nationally and globally, coinciding with the inclusion of population health within healthcare reform goals, suggests a role for AHCs in contributing to health system change and improvement. As population health is concerned with the health outcomes of a group of people and with the distribution of health outcomes within the group, patterns of health determinants and related policies and interventions, ${ }^{21}$ this role can be framed by the concept of health equity.

Health equity, as a concept, is defined as the absence of avoidable and unfair inequalities in health ${ }^{22}$ and can be differentiated from health inequalities or statistically described disparities alone by its moral and ethical dimension ('unfairness') - the determination of which involves examination of the inequalities in a social context. ${ }^{23}$ Equitable health systems, may thus be understood as those that actively ensure universal access to high quality health care, including establishing goals and initiatives to improve healthcare coverage across disadvantaged populations. ${ }^{24}$

Thus, the aim of this systematic review will be to review the literature on the role of AHCs in contributing to equitable health systems locally and globally. To achieve this aim, the review will address the following research questions sequentially:

1. How is health equity characterised, described and/ or operationalised in relation to AHC activity?

2. What are the drivers, barriers and facilitators of AHC activity relevant to health equity?

By positioning AHCs, in their health system context, as the unit of analysis, this review identifies AHCs as key health system structures that are being established or are undergoing transformation in a range of countries and settings and endeavours to shed light on whether they have a particular role to play in aiding efforts to build equitable health systems locally and globally. While significant diversity in AHC structures and their health system contexts is acknowledged, this review aims to identify any commonalities that might exist in published experiences and approaches, across different settings, of organisations identified as AHCs.

\section{METHODS AND ANALYSIS}

\section{Study design}

Efforts to encourage adoption of systematic review methodology within health policy research reflect its dominance in quantitative research fields. ${ }^{25}$ These efforts recognise the utility of systematic reviews as helpful tools enabling appraisal of complex findings from multiple disciplines and methods. ${ }^{26}$ Accordingly, a systematic methodology, following the online supplementary PRISMA 2009 checklist, ${ }^{*}$ has been identified as the method best able to address the research questions of this review, as well as to maximise reproducibility and confidence in the findings. The approach also recognises the increasingly acknowledged value of equity-focused systematic reviews as sources of evidence for healthcare and health systems decision making. ${ }^{22}$

Following the definition of 'systematic review' in the Preferred Reporting Items for Systematic Reviews and Meta-analyses for Protocols (PRISMA-P) guidelines, ${ }^{27}$ this protocol outlines the review objectives (aim and research questions), proposed systematic search methods meeting identified eligibility criteria, proposed assessment of quality and relevance of included studies and proposed

*http://www.prisma-statement.org/. 
Table 1 Inclusion and exclusion criteria

\begin{tabular}{lll}
\hline Criteria & Inclusion & Exclusion \\
\hline Time period & $2000-2016$ & Publications outside the inclusion period \\
Language & English & Materials not published in English \\
Literature type & Published peer-reviewed and grey literature & Clinical intervention or trial \\
& & Unpublished data \\
Research questions & Identifies AHCs as a unit of analysis & AHCs not a unit of analysis \\
& Addresses health equity concepts in relation to & Study does not address health equity \\
& AHConcepts in relation to AHC activity/ & role
\end{tabular}

AHCs, academic health centres.

approach to systematic synthesis of the characteristics and findings of the included studies. Consideration has also been given to the extensions for equity-focused reviews described in the PRISMA-Equity 2012 Extension. $^{\dagger}$ A preliminary search of the literature has informed the design of this review. Any updates and amendments to this protocol will be summarised in the final review manuscript.

\section{Eligibility criteria}

Reflecting the likelihood that valuable information may be found within non-peer-reviewed sources, all types of published peer-reviewed and grey (non-peer reviewed reports and other protocol materials) literature will be included in the review. Studies reporting clinical interventions or trials, however, will be excluded from the review as they are unlikely to supplement review findings.

In order to maximise contemporary policy relevance of the findings, literature searching will be limited to the time frame of 1 January 2000-31 December 2016. Literature on AHCs in any country will be included, although for pragmatic reasons the review will only include literature published in the English language. As the interest of the review is on the role of AHCs in contributing to equitable health systems, literature not explicitly addressing connection between AHCs and issues relevant to health equity will be excluded.

Table 1 summarises the inclusion and exclusion criteria that will define the scope and number of publications included in the review.

\section{Literature search}

\section{Search strategy}

For the purpose of the review, AHCs will include all organisations that self-identify or are identified by others as academic health/medical (science) centres/systems/ networks, integrated health research centres, advanced health research and translation centres and/or other proxy terms. While it is acknowledged that not all organisations that might objectively be defined as an AHC would self-identify as such, this operational definition was determined

\footnotetext{
${ }^{\dagger}$ http://prisma-statement.org/Extensions/Equity.aspx.
}

for pragmatic reasons and to enable assessment of the literature using these terms. Health equity will include all policies, programmes and objectives that aim to address inequalities in health that are avoidable yet not avoided. ${ }^{28}$ Implicit in this definition of health equity are concepts of addressing disadvantage and improving health for underserved populations, which include consideration of the social determinants of health. ${ }^{29}$

Drawing from these broad definitional parameters, search keywords will be derived using the pearl harvesting method as described by Sandieson et al. ${ }^{30}$ This will be undertaken in consultation with a university librarian with database and search strategy expertise. Boolean operators and truncated terms will be used to maximise the sensitivity and efficiency of the search strategy, and medical subject headings terms will be included where applicable. The search keywords will be piloted before the final list of search terms is selected.

\section{Databases}

A pilot list of bibliographic databases, below, was selected for its breadth of subject matter and likelihood of containing a wide range of study types. This list will be refined based on identification of duplication and expert consultation.

1. Google Scholar.

2. Scopus.

3. MEDLINE (Ovid).

4. PsycInfo (Ovid).

5. CINAHL (Ebsco).

6. ERIC.

7. ProQuest Dissertations \& Theses.

8. Cochrane Library.

9. Evidence Based Medicine (EBM) Reviews.

10. Campbell Library.

11. Informit health suite.

\section{Other data sources}

As systematic reviews of complex evidence have been shown to benefit from a range of search strategies, ${ }^{31}$ the searching strategy will also include snowballing (pursuing references of references), browsing of library shelves, asking colleagues and being alert to serendipitous discovery. In addition, direct contact will be made with 
authors or experts in the field in order to seek suggestions on additional literature sources relevant to the review, particularly grey literature.

\section{Study selection}

Search results will be uploaded into Endnote, combined and duplicates removed. Unique records will then enter the title-abstract screening stage. Following the 'single screening' method, ${ }^{32}$ one reviewer will screen and assign an 'included', 'provisionally included' or 'excluded' code to a title-abstract record, based on assessment of relevance to the research questions. Records deemed relevant to either question will be included. Two additional reviewers will be consulted where records are deemed 'provisionally included' by the first reviewer. Full-text papers will be accessed for the selected reviews. A PRISMA flow diagram will be used to report the results of this process.

\section{Data extraction}

Data from selected articles will be recorded in a locally developed data extraction form and independently validated by one reviewer. Standard information will be extracted on each paper, as well as data specific to the review questions. Data will be extracted against the following categories:

- full reference: including author names, year of publication and journal;

- country of origin: country of the study institution;

- study setting: country of the study subject;

- study type: for example, empirical research, policy paper, commentary, review;

- theoretical or conceptual perspective;

- link to equity agenda: short summary of nature of relevance to health equity agenda;

- quality and relevance assessment outcomes.

As there is significant variation in the contexts in which AHCs operate, which is likely to impact on AHC activity relevant to health equity, a separate table will list the AHCs identified in the included papers alongside key features of the health systems in which they operate. Health status statistics relevant to the locations of activity of these AHCs will also be listed in this table to identify key population characteristics. Information in both tables will be included in the analysis of the review findings.

\section{Quality and relevance assessment}

Quality and relevance appraisal of selected publications will involve two assessment components as described by Gough $e t a l^{25}$ : assessment of the study's relevance to the review questions and the quality of the execution of the methods employed by the study.

Relevance will be assessed qualitatively with reference to the inclusion/exclusion criteria, with highly relevant publications scoring 1 and less relevant publications scoring 0 . To assess quality, an initial appraisal will be undertaken of the level of evidence according to the Joanna Briggs Institute (JBI) criteria. ${ }^{33}$ Following this, studies will be assessed for methodological quality and approach to bias using the relevant JBI critical appraisal tools aligned with study type, such as the systematic review, qualitative or text and opinion checklists. ${ }^{34}$ Studies showing $50 \%$ or more compliance with the checklist will receive a score of 1 , with studies showing less than this scoring 0 .

The results of this appraisal will be recorded against each listing within the data extraction template and scores aggregated. Although it is not anticipated that any studies will be excluded from the review, findings demonstrating limited relevance and/or quality of studies will contribute to analysis of review limitations.

\section{Data analysis}

Data analysis will follow a thematic synthesis ${ }^{35}$ approach to enable the development of descriptive themes from the research. Analytic themes will then be generated through a process of interpretation and analysis. A narrative summary will interpret the results and describe how they relate to the review's aim and questions, with reference to the key contextual information obtained in the data extraction phase described above. Development of mid-range theory or a conceptual framework to better understand the roles and functions of AHCs will then be undertaken to aggregate the results into hypotheses for future research.

\section{ETHICS AND DISSEMINATION}

As the review is focused on the analysis of secondary data, it does not require ethics approval. The results of the study will be disseminated through articles in peer-reviewed journals and trade publications as well as presentations at relevant national and international conferences. Results will be further disseminated through networks and associations of AHCs, including the Association of Academic Health Centers International. It is also anticipated that this study will inform the activity and development of the Tropical Australian Academic Health Centre being established in northern Queensland, Australia. This study is being undertaken as part of a $\mathrm{PhD}$ thesis by the first author.

\section{DISCUSSION AND CONCLUSION}

To the best of our knowledge, only one global review using systematic methods (scoping review) has been undertaken to date of the literature on AHCs. ${ }^{1}$ This review assessed the literature exploring the managerial, political and cultural perspectives of AHCs, finding the literature on AHCs 'largely atheoretical and heavily dominated by case study reports from North America'. The need for more theoretically informed studies on AHCs, both within and across nations, has been previously identified. ${ }^{6}$ Building a conceptual framework to examine the role of AHCs in relation to broader healthcare goals, therefore, appears to fill an important gap in the literature.

The current review is one of the first attempts to synthesise and critically appraise evidence on the role of AHCs in contributing to equitable health systems locally and 
globally. Other strengths of this review include the use of the novel PRISMA-P guidelines and the relevant JBI critical appraisal tools.

Limiting our search to literature published in the English language may lead to the omission of studies from non-English speaking countries. Although it is difficult to predict in which cases the exclusion of studies published in languages other than English will bias review findings ${ }^{36}$ this review will also consider the possible effect of language bias in relation to the findings. Limiting the review to published papers only will also result in the omission of unpublished documentation of possible relevance to the review questions. It is also acknowledged that the search terms used to describe AHCs in the review may lead to the omission of activity of AHCs in countries that do not use these terms or that do not describe themselves as such even though they may fulfil an objective definition of an AHC. Finally, the results of the review may also be limited by the study designs of included studies, which are likely to be mostly observational studies and may present challenges in the quality assessment phase. These limitations and challenges will be considered in relation to the review findings.

AHC models are being adopted and adapted worldwide within health systems that are evolving alongside local and global reform and development agendas. As such, consideration of the intersection between the development and transformation of AHCs and broader activity to establish equitable health systems may clarify the purpose of AHCs and determine optimal AHC structures for meeting health needs of disadvantaged populations. This review may encourage policy makers to draw AHCs further into health system reform agendas as implementation vehicles, and offers utility to those involved in developing, leading and managing AHC activity across a range of countries.

Acknowledgements $\mathrm{AE}$ is a PhD candidate within the College of Public Health, Veterinary and Medicine Sciences, Division of Tropical Health and Medicine, James Cook University; SMT and JT are thesis supervisors; PVO is an external thesis advisor. The authors would like to thank the reviewers for their valuable input on the first version of this manuscript.

Contributors AE conceived and designed the study and drafted the manuscript. SMT, JT and PVO provided input into study design and helped revise the manuscript. All authors read and approved the final version of the manuscript.

Funding This research received no specific grant from any funding agency in the public, commercial or not-for-profit sectors. PVO is supported by the National Institute for Health Research (NIHR) Biomedical Research Centre, Oxford.

Competing interests AE is Program Operations Manager at the Tropical Australian Academic Health Centre, a developing alliance between the Cairns and Hinterland, Mackay, North West, Torres and Cape, and Townsville Hospital and Health Services; the Northern Queensland Primary Health Network; and James Cook University, including the Australian Institute of Tropical Health and Medicine.

Provenance and peer review Not commissioned; externally peer reviewed.

Open Access This is an Open Access article distributed in accordance with the Creative Commons Attribution Non Commercial (CC BY-NC 4.0) license, which permits others to distribute, remix, adapt, build upon this work non-commercially, and license their derivative works on different terms, provided the original work is properly cited and the use is non-commercial. See: http://creativecommons.org/ licenses/by-nc/4.0/ (c) Article author(s) (or their employer(s) unless otherwise stated in the text of the article) 2017. All rights reserved. No commercial use is permitted unless otherwise expressly granted.

\section{REFERENCES}

1. French CE, Ferlie E, Fulop NJ. The international spread of Academic Health Science Centres: a scoping review and the case of policy transfer to England. Health Policy 2014;117:382-91.

2. Wartman SA. Association of Academic Health Centers International. The Academic Health Center: Evolving Organizational Models. Issues Brief, 2007.

3. Culbertson RA, Goode LD, Dickler RM. Organizational models of medical school relationships to the clinical enterprise. Acad Med 1996;71:1258-74.

4. Weiner BJ, Culbertson R, Jones RF, et al. Organizational models for medical school-clinical enterprise relationships. Acad Med 2001;76:113-24.

5. Ovseiko PV, Davies SM, Buchan AM. Organizational models of emerging academic health science centers in England. Acad Med 2010;85:1282-9.

6. Davies SM, Tawfik-Shukor A, de Jonge B, Structure deJB. Structure, governance, and organizational dynamics of university medical centers in the Netherlands. Acad Med 2010;85:1091-7.

7. National Task Force on the Future of Canada's Academic Health Sciences Centres. Three missions one future: optimizing the perfomance of Canada's academic health sciences centres. Ottawa ON: Association of Canadian Academic Healthcare Organizations, 2010.

8. Fisk NM, Wesselingh SL, Beilby JJ, et al. Academic health science centres in Australia: let's get competitive. Med J Aust 2011;194:59-60.

9. Berwick DM, Nolan TW, Whittington J. The triple aim: care, health, and cost. Health Aff 2008;27:759-69.

10. Kirch DG, Ast CE. Health Care transformation: the role of Academic Health Centers and their psychologists. J Clin Psychol Med Settings 2016.

11. Wartman SA. The transformation of Academic Health Centers. Boston: Academic Press, 2015.

12. Rahn DW. Foreward. In: SA, . The Transformation of Academic Health Centers. Boston: Academic Press 2015 xv.xvii.

13. Jennings GL, Walsh MK. Integrated health research centres for Australia. Med J Aust 2013;199:320-1.

14. Gourevitch MN. Population health and the academic medical center: the time is right. Acad Med 2014;89:544-9.

15. Ackerly DC, Udayakumar K, Taber R, et al. Perspective: global medicine: opportunities and challenges for academic health science systems. Acad Med 2011;86:1093-9.

16. Dzau VJ, Ackerly DC, Sutton-Wallace P, et al. The role of academic health science systems in the transformation of medicine. Lancet 2010;375:949-53.

17. Michener L, Cook J, Ahmed SM, et al. Aligning the goals of community-engaged research: why and how academic health centers can successfully engage with communities to improve health. Acad Med 2012;87:285-91.

18. Golden RN, Drezner MK, Grossman JE, et al. Chapter 4 - The Changing Roles and Expectations of Faculty. Wartman SA, ed. The transformation of Academic Health Centers. Boston: Academic Press, 2015:29-37

19. DiSesa VJ, Kaiser LR. What's in a name? the necessary transformation of the Academic Medical Center in the era of Population Health and Accountable Care. Acad Med 2015;90:842-5.

20. Perman JA, Mullins CD, Newhouse R. Chapter 19 - Population Health and the Patient. Wartman SA, ed. The transformation of Academic Health Centers. Boston: Academic Press, 2015:195-202.

21. Kindig D, Stoddart G. What is population health? Am J Public Health 2003;93:380-3.

22 Welch V, Petticrew M, Petkovic J, et al. Extending the PRISMA statement to equity-focused systematic reviews (PRISMA-E 2012): explanation and elaboration. J Clin Epidemiol 2016;70:68-89.

23 Whitehead $M$. The concepts and principles of equity and health. Int $J$ Health Serv 1992;22:429-45.

24. Gwatkin DR, Bhuiya A, Victora CG. Making health systems more equitable. Lancet 2004;364:1273-80.

25. Gough D, Oliver S, Thomas J. Learning from research: systematic reviews for informing policy decisions, a quick guide. London: A paper for the Alliance for Useful Evidence, 2013. 
26. World Health Organisation. Systematic Reviews for Health Policy and Systems Research: Alliance for Health Policy and Systems Research. 2009.

27. Moher D, Shamseer L, Clarke M, et al. Preferred reporting items for systematic review and meta-analysis protocols (PRISMA-P) 2015 statement. Syst Rev 2015;4:1.

28. Marmot M; Commission on Social Determinants of Health. Achieving health equity: from root causes to fair outcomes. Lancet 2007;370:1153-63.

29. Marmot M, Friel S, Bell R, et al. Closing the gap in a generation: health equity through action on the social determinants of health. Lancet 2008;372:1661-9.

30. Sandieson RW, Kirkpatrick LC, Sandieson RM, et al. Harnessing the power of Education Research Databases with the Pearl-Harvesting Methodological Framework for Information Retrieval. J Spec Educ 2010;44:161-75.
31. Greenhalgh T, Robert G, Macfarlane F, et al. Storylines of research in diffusion of innovation: a meta-narrative approach to systematic review. Soc Sci Med 2005;61:417-30.

32. Shemilt I, Khan N, Park S, et al. Use of cost-effectiveness analysis to compare the efficiency of study identification methods in systematic reviews. Syst Rev 2016;5:1-13.

33. The Joanna Briggs Institute and the University of Adelaide. New JBI levels of evidence. Developed by the Joanna Briggs Institute Levels of Evidence and Grades of Recommendation Working Party. 2014.

34. The Joanna Briggs Institute and The University of Adelaide. Critical Appraisal Tools. 2016 http://joannabriggs.org/research/criticalappraisal-tools.html (accessed 9 Nov 2016).

35. Thomas J, Harden A. Methods for the thematic synthesis of qualitative research in systematic reviews. BMC Med Res Methodol 2008;8:45.

36. Higgins JPT, Green S. Section 10.2.2.4: Language bias. In: Higgins JPT, Green S, eds. Cochrane Handbook for Systematic Reviews of Interventions Version 510. The Cochrane Collaboration, 2011. 